Quim. Nova, Vol. 36, No. 5, 716-719, 2013

\title{
DETERMINAÇÃO ESPECTROFOTOMÉTRICA DE CLORETO EM CIMENTO APÓS PREPARO DE AMOSTRA POR PIROIDRÓLISE
}

\author{
Fabio A. Duarte e Ederson R. Pereira \\ Escola de Química e Alimentos, Universidade Federal do Rio Grande, 96203-900 Rio Grande - RS, Brasil \\ Eder L. M. Flores \\ Coordenação de Engenharia de Alimentos, Universidade Tecnológica Federal do Paraná, 85884-000 Medianeira - PR, Brasil \\ Edson I. Muller, Erico M. M. Flores e Valderi L. Dressler* \\ Departamento de Química, Universidade Federal de Santa Maria, 97105-900 Santa Maria - RS, Brasil
}

Recebido em 19/7/12; aceito em 24/11/12; publicado na web em 8/3/13

\begin{abstract}
SPECTROPHOTOMETRIC DETERMINATION OF CHLORIDE IN CEMENT AFTER SAMPLE PREPARATION BY PYROHYDROLYSIS. A method based on pyrohydrolysis was proposed for cement sample preparation and further chloride determination by spectrophotometry using flow injection analysis. Analytical parameters were evaluated and, under the selected conditions, the calibration curve was linear in the range of 0.2 to $10.0 \mu \mathrm{g} \mathrm{mL}^{-1}$ with $\mathrm{r}^{2}=0.998$. The limit of detection was $5 \mu \mathrm{g} \mathrm{g}^{-1}$ of chloride and the relative standard deviation was less than $7 \%$. The proposed pyrohydrolysis method is relatively simple and can be used for sample preparation for further spectrophotometric determination of low concentrations of chloride in cement.
\end{abstract}

Keywords: pyrohydrolysis; chloride; cement.

\section{INTRODUÇÃO}

Diversos são os efeitos causados pelo cloreto presente em cimento para construção civil, bem como na própria indústria produtora de cimento. Entretanto, um dos efeitos negativos mais conhecidos deste elemento é decorrente da sua característica corrosiva. Geralmente, a corrosão causada pelo cloreto em concreto leva ao seu inchamento e, consequentemente, à sua fragmentação. Uma vez fragmentado, há um aumento do contato do concreto e as estruturas metálicas com o ar e a umidade contendo dióxido de carbono, ${ }^{1}$ sulfato $^{2}$ e outros ânions ${ }^{3-5}$ propiciando um aumento da corrosão. ${ }^{6}$ Portanto, o teor de cloreto permissível no cimento deve estar dentro de determinados valores máximos. ${ }^{7,8}$ Neste contexto, na indústria de produção de cimento, o nível máximo estipulado para cloreto em cimento é de $150 \mu \mathrm{g} \mathrm{g}^{-1}$, uma vez que o mesmo volatiliza nas partes que operam a altas temperaturas e condensam em determinadas regiões frias do forno. $\mathrm{O}$ acúmulo do cloreto pode levar a sérios problemas de operação do forno, aumentando os custos de produção. ${ }^{9}$

Devido à influência do cloreto na durabilidade de materiais de concreto, o controle deste elemento em cimento pode assegurar a qualidade e segurança destas estruturas. ${ }^{10} \mathrm{O}$ método de referência usado para a determinação de cloreto em materiais à base de cimento é baseado na dissolução da amostra com ácido nítrico e posterior determinação pelo método de Volhard. ${ }^{11}$ Porém, este método não é adequado para determinar baixas concentrações de cloreto, bem como o ponto final é difícil de ser visualizado. ${ }^{8}$ Entretanto, outras técnicas empregadas para determinação de cloreto em cimento envolvem o uso de titulação potenciométrica, ${ }^{10}$ potenciometria com eletrodo íon seletivo (ISE), ${ }^{12}$ espectroscopia de emissão óptica com plasma induzido por laser (LIBS), ${ }^{13,14}$ cromatografia de íons (IC) ${ }^{15,16}$ e espectrometria de emissão óptica com plasma indutivamente acoplado (ICP OES). ${ }^{7}$ No entanto, estas técnicas estão sujeitas a interferências diversas e o preparo adequado da amostra é, em muitos casos, a etapa fundamental para que se obtenham resultados confiáveis.

\footnotetext{
*e-mail: vdressler@gmail.com
}

Entre os métodos tradicionalmente empregados para o preparo de amostras para a determinação de halogênios, destacam-se a fusão alcalina, ${ }^{17}$ a destilação (método de Willard e Winter), ${ }^{18}$ dissolução com ácidos ${ }^{8}$ e sistemas baseados na decomposição assistida por micro-ondas (MAD) ${ }^{19,20}$ e combustão iniciada por micro-ondas (MIC). ${ }^{21,22}$ Entretanto, estes métodos possuem algumas desvantagens como o grande número de etapas, longo tempo de preparo e custo relativamente elevado. Os métodos de fusão, por exemplo, não permitem a separação do analito da matriz, enquanto que com o uso de ácidos minerais podem ser formados e perdidos compostos voláteis do analito durante a etapa de preparo. ${ }^{23}$

Com o objetivo de minimizar estes efeitos, a piroidrólise pode ser considerada um método adequado para o preparo de amostras inorgânicas e biológicas para a posterior determinação de cloreto por diversas técnicas analíticas. ${ }^{17,23,24}$ Este método tem sido aplicado recentemente para diferentes matrizes ${ }^{23-25}$ e consiste na hidrólise de alguns elementos a elevadas temperaturas $\left(\sim 1000{ }^{\circ} \mathrm{C}\right)$ na presença de vapor d'água. Quando presentes, alguns elementos, tais como os halogênios, boro e enxofre são quantitativamente volatilizados da amostra na forma de seus correspondentes ácidos voláteis ou $\mathrm{SO}_{2} \mathrm{e}$ $\mathrm{SO}_{3}$, no caso do enxofre. Os compostos volatilizados são carreados até um condensador ${ }^{26}$ ou um recipiente contendo uma solução absorvedora $^{24}$ para a subsequente determinação. Como a solução resultante não contém muitos sólidos dissolvidos ou soluções ácidas concentradas, esta pode ser adequada para a análise direta. Desta forma, com a piroidrólise, o analito pode ser separado da matriz, minimizando assim possíveis interferências na etapa de determinação do analito, ${ }^{23}$ além de ser um método relativamente rápido e com baixo consumo de reagentes quando comparado ao método oficial (ASTM C114-11b, extração ácida e determinação pelo método de Volhard). ${ }^{11}$

Os métodos espectrofotométricos adaptados à análise por injeção em fluxo (FIA) ${ }^{27}$ permitiram um grande avanço no campo da mecanização em química analítica. De maneira geral, nestes sistemas a amostra é introduzida em um fluído carregador que permite seu transporte até o detector. Os sistemas FIA permitem proceder determinações mais rápidas do que métodos analíticos conduzidos de forma manual, principalmente onde é necessário a mistura de diversos 
reagentes, análises envolvendo reagentes tóxicos e de baixa estabilidade e procedimentos que envolvem etapas de pré-concentração e separação do analito durante o transporte da amostra. ${ }^{28,29}$ Além disso, possui como características intrínsecas a maior rapidez de análise, melhor repetitividade das medidas e a pequena quantidade de resíduo gerado. ${ }^{27}$

Neste sentido, o presente trabalho teve por objetivo avaliar o emprego da piroidrólise para o preparo de amostras de cimento do tipo Portland para posterior determinação espectrofotométrica de cloreto utilizando um sistema FIA. Foram estudadas condições referentes ao preparo da amostra por piroidrólise. A exatidão foi avaliada por meio da análise de materiais de referência certificados (CRM).

\section{PARTE EXPERIMENTAL}

\section{Instrumentação}

Para a determinação do cloreto foi empregado um sistema FIA, o qual é mostrado na Figura 1. Este sistema consiste de um injetor (comutador) construído em acrílico, uma bomba peristáltica (Ismatec, Suíça) de oito canais com velocidade variável, tubos de polietileno ( $0,8 \mathrm{~mm}$ de d.i.) e tubos de Tygon.

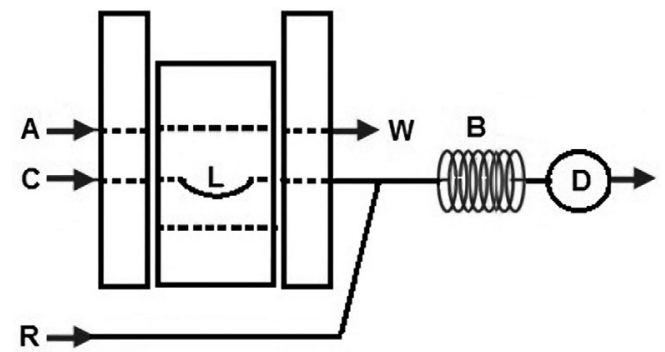

Figura 1. Sistema de injeção em fluxo e detecção espectrofotométrica (480 nm) para determinação de cloreto. A: amostra; $C$ : carreador; L: alça de amostragem (100 cm); B: bobina de reação $(70 \mathrm{~cm}) ; W:$ descarte; $R$ : reagentes (solução de 0,06\% $\mathrm{Hg}(\mathrm{SCN})_{2}+3 \mathrm{Fe}\left(\mathrm{NO}_{3}\right)_{3} .9 \mathrm{H}_{2} \mathrm{O}$ em $\mathrm{HNO}_{3} 2 \mathrm{~mol}$ $\left.L^{-1}\right)$; D: detector

As determinações de cloreto foram feitas em espectrofotômetro (Femto 432, São Paulo, Brasil) munido de cubeta de fluxo (Femto). O comprimento de onda foi fixado em $480 \mathrm{~nm}$. Os sinais de absorbância foram processados em altura de pico. A reação de piroidrólise foi feita em sistema desenvolvido no próprio laboratório, adaptado de trabalhos previamente descritos na literatura. ${ }^{23,26,30}$

\section{Reagentes}

A água utilizada foi previamente destilada, deionizada em uma coluna de troca iônica e, posteriormente, purificada em um sistema Milli-Q (Millipore, Bedford, EUA), com resistividade de 18,2 M $\Omega$ $\mathrm{cm}$. A descontaminação dos materiais utilizados foi feita com solução de $\mathrm{HNO}_{3} 10 \%$ (v/v), preparada a partir de $\mathrm{HNO}_{3} 65 \%(\mathrm{~m} / \mathrm{m})($ Carlo Erba, Milão, Itália), o qual foi purificado em sistema de destilação abaixo do ponto de ebulição (modelo duoPUR 2.01E, Millestone, Sorisole, Itália). Os materiais utilizados para o preparo e armazenamento das soluções foram imersos em solução de $\mathrm{HNO}_{3} 10 \%$ (v/v) por, pelo menos, $24 \mathrm{~h}$ e depois lavados com água. As soluções de calibração foram preparadas a partir de uma solução estoque de cloreto (1000 $\mathrm{mg} \mathrm{L}^{-1}$, preparada a partir da dissolução de $\mathrm{NaCl}$ - Merck, Darmstadt, Alemanha) em água. $\mathrm{O}$ pentóxido de vanádio $\left(\mathrm{V}_{2} \mathrm{O}_{5}\right)$ utilizado na piroidrólise foi preparado de acordo com Nebesar, ${ }^{31} \mathrm{a}$ partir de $\mathrm{NH}_{4} \mathrm{VO}_{3}$ (Merck), mediante aquecimento em forno mufla a $500{ }^{\circ} \mathrm{C}$ durante $4 \mathrm{~h}$.

\section{Amostras}

Amostras de cimento foram adquiridas no comércio local: duas amostras de cimento Portland pozolânico (1 e 2) de diferentes fabricantes e duas amostras de cimento Portland de alta resistência inicial ( 3 e 4 ) de diferentes fabricantes. As amostras foram secas em estufa a $60{ }^{\circ} \mathrm{C}$ até peso constante e, posteriormente, homogeneizadas. A granulometria era inferior a $100 \mu \mathrm{m}$.

Todas as otimizações foram feitas com CRM de origem geológica (JR-1 do Geological Survey of Japan) com concentração de cloreto de $920 \pm 81 \mu \mathrm{g} \mathrm{g}^{-1}$. A exatidão do método foi avaliada utilizando CRM de origem geológica (JGb-1, JR-1 e JR-2 do Geological Survey of Japan) e de sedimento marinho (PACS-2, HISS- 1 e MESS-2, National Research Council of Canada). Apesar da matriz destes CRMs serem de composição diferente à das amostras, estes foram utilizados tendo-se em vista que não há CRM de cimento com concentração certificada de cloreto.

\section{Piroidrólise e determinação de cloreto em cimento}

O procedimento de piroidrólise empregado neste estudo foi adaptado do procedimento descrito por Dressler e colaboradores. ${ }^{26}$ Para tal, alíquotas de $50 \mathrm{mg}$ de amostra previamente misturadas com $\mathrm{o}_{2} \mathrm{O}_{5}$ foram pesadas diretamente no suporte de quartzo, o qual foi introduzido no interior do reator. $\mathrm{O}$ frasco coletor, contendo $5 \mathrm{~mL}$ de água como solução absorvedora, foi conectado no capilar na saída do condensador e a vazão do gás carregador (ar) foi ajustada, cuja finalidade é a de transportar o vapor d'água contendo o analito através do sistema de piroidrólise. A seguir, foi iniciado o aquecimento do reator e do gerador de vapor d'água. Para o aquecimento do reator, foi utilizado um forno eletrotérmico, cuja temperatura máxima é de $1200{ }^{\circ} \mathrm{C}$. Após a reação de piroidrólise, o frasco coletor foi removido e o volume completado para $10 \mathrm{~mL}$ com água. A influência da vazão de ar (entre 0,1 e 1,0 $\mathrm{L} \mathrm{min}^{-1}$ ), a temperatura do reator de piroidrólise, a relação entre a massa do $\mathrm{V}_{2} \mathrm{O}_{5}$ e da amostra (entre 0 e 5), a vazão do gás carregador (entre 0,05 e $0,5 \mathrm{~L} \mathrm{~min}^{-1}$ ), o tempo de piroidrólise (entre 2,5 e $15 \mathrm{~min}$ ) e a quantidade de amostra (entre 5 e $100 \mathrm{mg}$ ) foram avaliadas de forma a se obter a máxima eficiência do sistema de preparo da amostra.

A determinação espectrofotométrica de cloreto com auxílio do sistema FIA foi feita segundo Iwasaki e colaboradores. ${ }^{32}$ A determinação do cloreto foi feita indiretamente (Reação 1), a partir da formação do complexo $\mathrm{Fe}(\mathrm{SCN})^{2+}$ (Reação 2), de coloração vermelha.

$$
\begin{aligned}
\mathrm{Hg}(\mathrm{SCN})_{2}+2 \mathrm{Cl}^{-} & \leftrightarrow \mathrm{HgCl}_{2}+2 \mathrm{SCN}^{-} \\
\mathrm{Fe}^{3+}+\mathrm{SCN}^{-} & \leftrightarrow \mathrm{Fe}(\mathrm{SCN})^{2+}
\end{aligned}
$$

A vazão da amostra foi fixada em $2,3 \mathrm{~mL} \mathrm{~min}^{-1}$ e a vazão da água utilizada como solução carreadora foi fixada em $4,7 \mathrm{~mL} \mathrm{~min}{ }^{-1}$. Como reagente colorimétrico foi utilizada uma mistura de $\mathrm{Hg}(\mathrm{SCN})_{2}$ e Fe( $\left(\mathrm{NO}_{3}\right)_{3} \cdot 9 \mathrm{H}_{2} \mathrm{O}$ em meio de $\mathrm{HNO}_{3} 2 \mathrm{~mol} \mathrm{~L}^{-1}$, cuja vazão da solução foi fixada em $1,0 \mathrm{~mL} \mathrm{~min}^{-1}$.

\section{RESULTADOS E DISCUSSÃO}

\section{Avaliação dos parâmetros que influenciam na piroidrólise}

Verificou-se que a vazão do ar, utilizado como carregador do vapor contendo o analito até o frasco coletor, de até $0,5 \mathrm{~L} \mathrm{~min}^{-1}$ não influencia na temperatura no interior do reator. Nestes casos, a temperatura permaneceu praticamente constante, variando entre 1050 e $1170{ }^{\circ} \mathrm{C}$. Desta forma, a vazão máxima do gás carregador para a recuperação quantitativa de cloreto foi fixada em $0,5 \mathrm{~L} \mathrm{~min}^{-1}$. Nesta condição, a 
temperatura é em torno de $1100^{\circ} \mathrm{C}$. Estas condições foram utilizadas para a otimização dos demais parâmetros.

Um auxiliar pode ser necessário na reação de piroidrólise, principalmente para o preparo de amostras de composição predominantemente inorgânica, onde os analitos podem estar presentes na forma de compostos refratários. No caso do cloreto, a presença do catalisador é necessária para a reação de piroidrólise, pois facilita a sua volatilização, provavelmente na forma de $\mathrm{HCl}$ gasoso. $\mathrm{O}$ uso do $\mathrm{V}_{2} \mathrm{O}_{5}$ foi avaliado devido ao baixo custo e ao bom desempenho na reação de piroidrólise quando comparado com outros catalisadores como o $\mathrm{U}_{3} \mathrm{O}_{8}$ e $\mathrm{WO}_{3}$, também recomendados para esta finalidade. Empregando proporções $\left(\mathrm{V}_{2} \mathrm{O}_{5}\right.$ :amostra) entre 0:1 e 1:1, as recuperações foram inferiores a $46 \%$. A partir da proporção de $3: 1$, a recuperação de cloreto é superior a $95 \%$, permanecendo constante até a proporção de 5:1. A proporção de 3:1 foi então escolhida para os próximos experimentos. Resultados semelhantes utilizando $\mathrm{V}_{2} \mathrm{O}_{5}$ como catalisador para a determinação de cloreto em diferentes materiais estão descritos na literatura. ${ }^{17,23}$

A vazão do gás carregador deve ser suficiente para permitir o transporte adequado do vapor d'água gerado através do sistema, transportando os produtos gasosos da piroidrólise até o condensador e o frasco coletor. Se a vazão for muito elevada pode haver o transporte excessivo de vapor d'água para o reator, diminuindo a temperatura no interior deste e dificultando a absorção do analito na solução absorvedora. Dessa forma, a influência da vazão de ar foi avaliada, considerando seu efeito na recuperação de cloreto. Para vazões de até $0,2 \mathrm{~L} \mathrm{~min}^{-1}$, não foram obtidas recuperações satisfatórias, sendo inferiores a $80 \%$ e os valores de desvio padrão relativo (RSD) superiores a $8 \%$. A partir de vazões de $0,3 \mathrm{~L} \mathrm{~min}^{-1}$, a recuperação de cloreto foi constante e superior a $98 \%$. Portanto, $0,4 \mathrm{~L} \mathrm{~min}^{-1}$ foi a vazão selecionada para a avaliação dos demais parâmetros.

O tempo de reação deve ser suficiente para a liberação completa do analito da matriz da amostra e, também, para garantir que todo o analito seja transportado para o frasco coletor, prevenindo efeitos de memória no sistema ou recuperação não quantitativa do analito. Após 7,5 min de reação, recuperações de cloreto superiores a $98 \%$ são observadas, permanecendo constantes até $15 \mathrm{~min}$. No entanto, optou-se em trabalhar com tempo de reação de $10 \mathrm{~min}$, condição que permite a recuperação quantitativa do cloreto e sem efeito de memória no sistema (avaliado através da análise de um branco após o CRM). O tempo de processamento da amostra pelo método proposto está de acordo com trabalhos reportados na literatura, ${ }^{23,25,26}$ que, geralmente, está entre 7 e 20 min de reação.

A influência da massa de amostra foi avaliada entre 5 e $100 \mathrm{mg}$, utilizando as condições previamente estabelecidas. Observou-se que, independente da massa de amostra, a recuperação de cloreto sempre foi superior a $98 \%$. Não foi possível verificar o comportamento do método com massas de amostra superiores a $100 \mathrm{mg}$, devido à limitação da dimensão do reator empregado neste trabalho, o qual não permite o uso de um suporte com capacidade para maior quantidade de amostra. Após a reação, combinando-se o tempo de piroidrólise de 10 min e vazão de ar de $0,4 \mathrm{~L} \mathrm{~min}^{-1}$, obtém-se um volume de solução condensada de aproximadamente $8 \mathrm{~mL}$, a qual foi aferida a $10 \mathrm{~mL}$ para posterior determinação de cloreto. Na Tabela 1 é mostrado um resumo das características do sistema de piroidrólise.

\section{Avaliação da exatidão, precisão e linearidade do método}

Para avaliar a exatidão do método foram analisados diferentes CRMs, cujos resultados estão mostrados na Tabela 2. De maneira geral, os resultados foram concordantes (superiores a 90\%) com os valores certificados dos CRMs.

A precisão do método, expressa como RSD, foi entre 6 e $10 \%$.
Tabela 1. Parâmetros estabelecidos para o sistema de piroidrólise e subsequente determinação espectrofotométrica de cloreto

\begin{tabular}{lc}
\hline Parâmetros & Resultados \\
\hline Vazão de ar, $\mathrm{L} \mathrm{min}^{-1}$ & 0,4 \\
Temperatura do reator, ${ }^{\circ} \mathrm{C}$ & 1100 \\
Tempo de reação, min & 10 \\
Massa de amostra, mg & $5-100$ \\
$\mathrm{~V}_{2} \mathrm{O}_{5}:$ amostra & $3: 1$ \\
Volume de solução final, $\mathrm{mL}$ & 10 \\
Determinações, $\mathrm{h}^{-1}$ & 5 \\
\hline
\end{tabular}

Tabela 2. Determinação de cloreto em materiais de referência certificados (CRM) utilizando piroidrólise (resultados em $\mu \mathrm{g} \mathrm{g}^{-1}$, correspondente à média \pm desvio padrão, $\mathrm{n}=3$ )

\begin{tabular}{cccc}
\hline CRM & $\begin{array}{c}\text { Valor de } \\
\text { referência }\end{array}$ & $\begin{array}{c}\text { Valor } \\
\text { determinado }\end{array}$ & $\begin{array}{c}\text { Concordância } \\
(\%)\end{array}$ \\
\hline HISS-1 & 3500 & $3300 \pm 200$ & 94 \\
MESS-2 & - & $15000 \pm 1000$ & - \\
PACS-2 & 30000 & $32700 \pm 2500$ & 109 \\
JGb-1 & 81 & $73 \pm 7$ & 90 \\
JR-1 & $920 \pm 81$ & $954 \pm 84$ & 104 \\
JR-2 & 736 & $737 \pm 53$ & 100 \\
\hline
\end{tabular}

Cabe destacar que na determinação espectrofotométrica de cloreto, os limites de detecção e quantificação do método (LOD e LOQ, 3 e 10 $\sigma$, respectivamente, $\mathrm{n}=10$ ) foram de 5 e $16 \mu \mathrm{g} \mathrm{g}^{-1}$ (considerando 100 $\mathrm{mg}$ de amostra e $10 \mathrm{~mL}$ de solução final), respectivamente. A faixa linear de calibração foi de 0,2 a $10 \mathrm{mg} \mathrm{L}^{-1}$ de cloreto e a sensibilidade do método foi considerada boa, se comparado com outros métodos de determinação. ${ }^{12,13,15,33}$ Cabe destacar que, com o aumento da massa de amostra e uso de IC com detector por condutividade, é possível reduzir o LOD para até $0,3 \mu \mathrm{g} \mathrm{g}^{-1} \cdot{ }^{16}$ Entretanto, como a concentração de cloreto em cimento é, na maioria dos casos, superior a $20 \mu \mathrm{g} \mathrm{g}^{-1},{ }^{8,16}$ o método de preparo da amostra utilizando piroidrólise é adequado para a determinação espectrofotométrica de cloreto em cimento.

\section{Aplicabilidade}

O método proposto foi aplicado para diferentes amostras de cimento Portland e os resultados estão mostrados na Tabela 3. Para amostras de cimento do tipo CP-IV, as concentrações são inferiores a $464 \pm 54 \mu \mathrm{g} \mathrm{g}^{-1}$, enquanto para amostras de cimento CP-V-ARI as concentrações são inferiores a $147 \pm 5 \mu \mathrm{g} \mathrm{g}^{-1}$, com RSD variando entre 3 e $14 \%$.

Tabela 3. Resultados obtidos para a determinação de cloreto em cimento (resultados em $\mu \mathrm{g} \mathrm{g}^{-1}$, correspondente à média \pm desvio padrão, $\mathrm{n}=3$ )

\begin{tabular}{cc}
\hline Amostras & Valor determinado \\
\hline CP-IV-32 a & $464 \pm 54$ \\
CP-IV-32 b & $127 \pm 13$ \\
CP-V-ARI a & $132 \pm 19$ \\
CP-V-ARI b & $147 \pm 5$ \\
\hline
\end{tabular}

As concentrações de cloreto determinadas nas diferentes amostras de cimento foram abaixo dos limites máximos permitidos por diversas normas, que consideram, em geral, $4000 \mu \mathrm{g} \mathrm{g}^{-1}$ de cloreto em 
peso seco de cimento. Para os Estados Unidos, a Federal Highway Administration (FHWA) estabelece limites menores do que 3000 $\mu \mathrm{g} \mathrm{g}^{-1}$ de cloreto em peso seco de cimento. ${ }^{34}$ No Brasil, entretanto, a Associação Brasileira de Normas Técnicas (ABNT) não reporta limites máximos de cloreto em cimento.

Potgieter e Panicheva ${ }^{8}$ analisaram 5 amostras de cimento e agregados de cimento. Método de Volhard e titulação potenciométrica foram avaliados para a determinação de cloreto, enquanto que, como preparo de amostra, três métodos de dissolução foram comparados. De maneira geral, foi mostrado que há uma grande variabilidade entre os diferentes procedimentos avaliados para a determinação de cloreto, sendo que os melhores resultados foram obtidos com extração ácida utilizando ácido acético e titulação potenciométrica para a determinação de cloreto. Ainda, segundo esse trabalho, o método de Volhard apresenta alguns inconvenientes que podem comprometer a precisão e exatidão dos resultados, como a coloração característica dos extratos de algumas amostras, que dificulta a visualização do ponto final da titulação.

\section{CONCLUSÕES}

A partir dos resultados obtidos pode-se afirmar que a piroidrólise é adequada para o preparo de amostras de cimento para posterior determinação espectrofotométrica de cloreto. A piroidrólise pode ser considerada uma alternativa viável frente às limitações dos métodos existentes para o preparo de amostras para a determinação de cloreto. $\mathrm{O}$ método desenvolvido é relativamente rápido e envolve baixo consumo de reagentes quando comparado ao método oficial (extração ácida e determinação pelo método de Volhard). O sistema de piroidrólise é de fácil montagem e operação, além de ser de baixo custo. Adicionalmente, a piroidrólise, devido à separação da matriz, minimiza possíveis interferências na etapa de determinação do analito, é desprovida de etapas de filtração e não depende da visualização de cor pelo analista. Portanto, pode ser considerado um método adequado para análises de rotina, principalmente se combinado com sistemas FIA, permitindo uma maior mecanização da análise.

\section{AGRADECIMENTOS}

À Fundação de Amparo à Pesquisa do Estado do Rio Grande do Sul (FAPERGS), ao Conselho Nacional de Desenvolvimento Tecnológico (CNPq) e Coordenação de Aperfeiçoamento de Nível Superior (CAPES).

\section{REFERÊNCIAS}

1. Kauer, J. A.; Freeman, R. L.; ACI Mater. J. 1955, 52, 447.

2. Corral-Higuera, R.; Arredondo-Rea, S. P.; Neri-Flores, M. A.; GómezSoberón, J. M.; Almeraya, C. F.; Castorena-González, J. H.; AlmaralSánchez, J. L.; Int. J. Electrochem. Sci. 2011, 6, 613.

3. Costa, A.; Appleton, J.; Mater. Struct. 1999, 32, 252.

4. Gjorv, Ø. E.; Vennesland, Ø.; Cement Concrete Res. 1979, 9, 229.

5. Song, H.-W.; Lee, C.-H.; Ann, K. Y.; Cement Concrete Comp. 2008, 30, 113.
6. Hope, B. B.; Page, J. A.; Poland, J. S.; Cement Concrete Res. 1985, 15, 863.

7. Potgieter, S. S.; Marjanovic, L.; Cement Concrete Res. 2007, 37, 1172.

8. Potgieter, S. S.; Potgieter, J. H.; Panicheva, S.; Mater. Struct. 2004, 37, 155.

9. Addis, B. J., ed.; Fulton's Concrete Technology, Portland Cement Institute: Midrand, 1994, p. 67.

10. Climent, M. A.; de Vera, G.; Viqueira, E.; López-Atalaya, M. M.; $C e$ ment Concrete Res. 2004, 34, 2291.

11. American Society of Testing and Materials; ASTM C114-11b: Standard Test Methods for Chemical Analysis of Hydraulic Cement, West Conshocken, 1934, reaprovado em 2011.

12. Angst, U.; Elsener, B.; Larsen, C. K.; Vennesland, Ø.; J. Appl. Electrochem. 2009, 40, 561.

13. Gehlen, C. D.; Wiens, E.; Noll, R.; Wilsch, G.; Reichling, K.; Spectrochim. Acta, Part B 2009, 64, 1135.

14. Weritz, F.; Schaurich, D.; Taffe, A.; Wilsch, G.; Anal. Bioanal. Chem. 2006, 385, 248.

15. Zanella, R.; Primel, E. G.; Martins, A. F.; J. Sep. Sci. 2001, 24, 230.

16. Noguchi, Y.; Zhang, L.; Maruta, T.; Yamane, T.; Kiba, N.; Anal. Chim. Acta 2009, 640, 106.

17. Wang, Q.; Makishima, A.; Nakamura, E.; Geostand. Geoanal. Res. 2009, 34, 175 .

18. Willard, H. H.; Arbor, A.; Winter, B.; Ind. Eng. Chem. Res. 1933, $5,7$.

19. Pereira, E. R.; Soares, B. M.; Vieira, J. P.; Mai, A. C. G.; Picoloto, R. S.; Müller, E. I.; Flores, E. M. M.; Duarte, F. A.; J. Braz. Chem. Soc. 2012, 23,846 .

20. Soares, B. M.; Vieira, A. A.; Lemões, J. S.; Santos, C. M. M.; Mesko, M. F.; Primel, E. G.; Montes D’Oca, M. G.; Duarte, F. A.; Bioresour. Technol. 2012, 110, 730.

21. Antes, F. G.; Duarte, F. A.; Mesko, M. F.; Nunes, M. A. G.; Pereira, V. A.; Müller, E. I.; Dressler, V. L.; Flores, E. M. M.; Talanta 2010, 83, 364.

22. Flores, E. M. M.; Mesko, M. F.; Moraes, D. P.; Pereira, J. S. F.; Mello, P. A.; Barin, J. S.; Knapp, G.; Anal. Chem. 2008, 80, 1865.

23. Antes, F. G.; Duarte, F. A.; Flores, E. L. M.; Paniz, J. N. G.; Flores, E. M. M.; Dressler, V. L.; Quim. Nova 2010, 33, 1130.

24. Antes, F. G.; Duarte, F. A.; Paniz, J. N. G.; Santos, M. F. P.; Guimarães, R. C. L.; Flores, E. M. M.; Dressler, V. L.; At. Spectrosc. 2008, 29, 157.

25. Taflik, T.; Duarte, F. A.; Flores, E. L. M.; Antes, F. G.; Paniz, J. N. G.; Flores, E. M. M.; Dressler, V. L.; J. Braz. Chem. Soc. 2012, 23, 488.

26. Dressler, V. L.; Pozebon, D.; Flores, E. L. M.; Paniz, J. N. G.; Flores, E. M. M.; Anal. Chim. Acta 2002, 466, 117.

27. Reis, B. F.; Giné, M. F.; Kronka, E. A. M.; Quim. Nova 1989, 12, 82.

28. Miranda, C. E. S.; Carrilho, E.; Quim. Nova 2002, 25, 412.

29. Ruzicka, J.; Hansen, E. H.; Anal. Chem. 2000, 72, 213.

30. Dressler, V. L.; Pozebon, D.; Flores, E. L. M.; Paniz, J. N. G.; Flores, E. M. M.; J. Braz. Chem. Soc. 2003, 14, 334.

31. Nebesar, B.; Talanta 1977, 25, 185

32. Iwasaki, I.; Utsumi, S.; Ozawa, T.; Bull. Chem. Soc. Jpn. 1952, 25, 226.

33. Junsomboon, J.; Jakmunee, J.; Talanta 2008, 76, 365.

34. Hansson, C. M.; Poursaee, A.; Jaffer, S. J.; Portland Cement Association, Skokie, 2007, p. 33. 